\title{
Educación rural y equidad en el periodo de confinamiento por la COVID-19: el caso de una escuela primaria multigrado
}

\author{
Equity in rural education during confinement by COVID-19: the case of a \\ multigrade primary school
}

\author{
Ana Laura González Lira ${ }^{a}$, Javier Moreno Tapia ${ }^{b}$
}

\begin{abstract}
:
This paper presents the results of a research realized in a rural primary school during confinement by COVID-19 pandemic. The main porpoise of this study was to analyze some difficulties in this context manly on the academic activities. We tried to understand the perspective of the teacher and the analysis of information from various sources. During the development of this report, we recovered published knowledge about multigrade schools during the pandemic. All this, based on the approach of educational equity and the advances in legislation in Mexico join information national and local. We applied interviews and questionnaires with a teacher in this case study. The results show that the current pandemic accentuates the social inequalities of the populations of students in the rural sector, in three components: access, permanence and educational achievement, a situation that marks the need for the design and implementation of equity policies and the importance of the scientific approach to the subject from educational research.
\end{abstract}

Keywords:

Rural schools, educational equity, multigrade

\section{Resumen:}

Este trabajo presenta los resultados de una investigación realizada en una escuela primaria rural durante el periodo de confinamiento derivado de la contingencia sanitaria por la pandemia de COVID-19. Su objetivo principal es analizar algunas dificultades que se presentan en el contexto de estudio para la continuidad de las actividades académicas, destacando la perspectiva del docente y el análisis de información proveniente de diversas fuentes. Se presenta un estado del conocimiento que integra investigaciones realizadas en el contexto de las escuelas multigrado y en el periodo de la pandemia. El trabajo se sustenta en el enfoque de equidad educativa y en los principios de la teoría sociocultural. Se recuperan los avances en materia de legislación educativa en México, y se contrastan con algunos datos de fuentes oficiales a nivel nacional y local, así como con información proveniente de la aplicación de instrumentos a docentes participantes en la investigación, realizada mediante una metodología de estudio de caso. Los resultados muestran que la actual pandemia acentúa las desigualdades sociales de los alumnos del sector rural, en tres componentes: acceso, permanencia y logro educativo, situación que marca la necesidad del diseño e implementación de políticas de equidad y la importancia de la aproximación científica al tema desde la investigación educativa.

\section{Palabras Clave:}

Escuelas rurales, equidad educativa, multigrado

\section{Introducción}

La investigación educativa asociada al periodo de confinamiento por la pandemia de COVID-19 se encuentra en ciernes. Los trabajos académicos publicados y consultados para la construcción del presente trabajo, se desarrollan básicamente desde dos líneas de análisis: 1) las dificultades relacionadas al contexto socioeconómico de los alumnos, que limitan el acceso a bienes y servicios mínimos para dar continuidad del ciclo escolar desde la modalidad a distancia y, 2) la precaria formación tecnológica de alumnos, padres de familia y docentes

\footnotetext{
${ }^{a}$ Secretaría de Educación Pública de Hidalgo, México. ORCID: https://orcid.org/0000-0003-2559-4004 Email: laupretty13@gmail.com

b Autor de Correspondencia, Universidad Autónoma del Estado de Hidalgo, México. ORCID: https://orcid.org/0000-0003-4029-5440 , Email: javier_moreno@uaeh.edu.mx
} 
(Carla, et al., 2020; Vivanco, 2020; Acero, et al., 2020; Mérida y Acuña, 2020; Gutiérrez, 2020; Loyd, 2020; TrejoQuintana, 2020; Amador, 2020; Didriksson, 2020; Hurtado, 2020).

En el ámbito nacional mexicano, la puesta en marcha del Programa Aprende en Casa, como mecanismo paliativo que busca dar seguimiento a las actividades académicas del ciclo escolar en los niveles de educación básica, ha derivado en múltiples críticas relacionadas a su factibilidad, pertinencia y eficacia en los contextos heterogéneos y desiguales que configuran el sistema educativo. Algunas investigaciones han demostrado que usar sólo sesiones remotas se contrapone a las realidades poco favorables y marginadas de las regiones del país (Mérida y Acuña, 2020; Gutiérrez, 2020; Loyd, 2020; Didriksson, 2020).

Bajo la premisa de que proseguir el ciclo escolar a través de las clases en línea supone contar con dispositivos electrónicos y servicios de red eléctrica y conexión a internet, las clases virtuales son accesibles para una minoría de población rural (Carla, et al., 2020; Didriksson, 2020).

Ante este escenario, algunos docentes que laboran en comunidades rurales, derivado de la falta de conectividad, equipamiento e infraestructura tecnológica, han tenido que acudir personalmente a la localidad a entregar las fichas didácticas y materiales de forma impresa que promuevan el cumplimiento de los propósitos de aprendizaje, enmarcados en los planes y programas de estudio (Carla, et al., 2020; Gutiérrez, 2020). Si bien, ha sido una opción viable para que los estudiantes den continuidad a las actividades académicas, representa la acumulación de trabajos y tareas para docentes y padres de familia, así como el aumento de riesgo de contagio. Sin embargo, con esta labor los docentes no sólo intentan dar cumplimiento a un programa de estudios, sino también "sostener las relaciones escolares de una comunidad que por el momento no se puede reencontrar" (Gutiérrez, 2020: 3).

Por otro lado, las posibilidades de intervención de los padres de familia y tutores para acompañar los procesos formativos de los estudiantes están supeditadas por el nivel académico, el tiempo disponible, la alfabetización digital, así como sus actividades laborales y del hogar. Todas estas condiciones representan dificultades para involucrarse en las actividades escolares de los alumnos, pues además se requiere del conocimiento previo de los contenidos y su didáctica (Loyd, 2020; Carla, et al., 2020; Gutiérrez, 2020; Narváez y Yépez, 2021).

Asimismo, algunos autores refieren que, aun superando las dificultades de infraestructura tecnológica y de acceso a dispositivos electrónicos, es primordial comprender que las intencionalidades y lógicas formativas de la modalidad presencial, a distancia y virtual son diferentes (Coll y Monereo, 2008). Esto ha representado un complejo reto y evidenció la insuficiente y soslayada formación didáctica del profesorado con respecto a la creación de ambientes de aprendizaje mediados por las Tecnologías de la Información y la Comunicación (TIC) (Loyd, 2020; Vivanco, 2020; Gallardo, 2020).

Respecto al contexto de las escuelas primarias multigrado, los estudios que anteceden a este, han evidenciado que son instituciones que se localizan en su gran mayoría, en pequeñas comunidades de alta o muy alta marginación, que operan con precariedad en el acceso a servicios básicos y el equipamiento, la infraestructura, los materiales, además de que la formación docente está lejos de ser adecuados para el logro de aprendizajes (Arteaga, 2011; Estrada, 2015; Juárez, 2017; Aguilera et al., 2019; Rockwell y Garay, 2014; Weiss, 2000; Priego y Castro, 2021).

Una mirada retrospectiva a las escuelas de organización multigrado permite reconocer que los docentes y alumnos han enfrentado, a lo largo de la historia del Sistema Educativo Nacional, serios problemas para el cumplimiento de los objetivos educativos (Rockwell y Garay, 2014; Weiss, 2000; Estrada, 2015). El escenario emergente de la actual pandemia, ha representado nuevos desafíos para los diferentes actores educativos y el panorama resulta aún más desalentador, pues la precariedad del acceso a bienes y servicios tecnológicos limita la comunicación y la posibilidad de seguimiento de las actividades educativas.

Algunos estudios, por su parte, han centrado sus objetivos en la identificación de las ventajas pedagógicas que ofrece la coexistencia de alumnos de diferentes edades y grados escolares en una misma aula de clases. Se destaca la mejora de las relaciones sociales entre estudiantes, el trabajo cooperativo y colaborativo, la circulación de saberes, el desarrollo de la autonomía y la convivencia. En el aula multigrado, los alumnos cuentan con una variedad de ayudas, que no sólo provienen del docente, sino de la interacción espontánea con otros alumnos. Respecto a la enseñanza, los docentes reconocen como aspectos favorables la posibilidad de atención individualizada, el trabajo transversal con contenidos, la participación activa de los padres de familia en las actividades escolares, además de referir percepciones de libertad, autonomía y crecimiento profesional (Bustos, 2010; Santos, 2011).

Mediante el análisis de la equidad sobre las acciones públicas dirigidas a las escuelas multigrado en México, Priego y Castro (2019) refieren que existe vulneración del derecho de las Niñas, Niños y Adolescentes que viven en comunidades aisladas, ya que la política accionada es inequitativa y poco pertinente. En este sentido, las 
escuelas multigrado se caracterizan por tres dimensiones básicas, que han sido soslayadas dentro de la política educativa: la primera es la pedagógica-didáctica referida al trabajo con diversidad de contenidos y estudiantes; la segunda es la institucional-organizacional que involucra la gestión escolar y las actividades administrativas y la tercera, es la contextual, relacionada con elementos demográficos y socioeconómicos (INEE, 2019 cit. en Castro y Priego, 2019).

Este trabajo tiene como propósito presentar los resultados de una investigación que tuvo como objetivo principal, analizar algunas dificultades que se presentan en una escuela primaria rural para continuar las actividades académicas durante el periodo del confinamiento. Se destaca la perspectiva de un docente, el análisis de información basado en la triangulación de diversas fuentes y la presentación de los resultados a la luz del enfoque de equidad educativa y de la teoría sociocultural.

\section{Aproximación conceptual}

El enfoque de equidad educativa ha cobrado protagonismo en el ámbito de la educación en los últimos años, por lo que resulta fundamental una aproximación conceptual como punto de partida para discutir sus alcances en la realidad educativa en contextos específicos. Para efectos de este trabajo se recupera el concepto de Sánchez-Santamaría y Ballester (2013) quienes refieren que la equidad educativa es "un estado del proceso educativo basado en condiciones, experiencias y situaciones pedagógicas orientadas al éxito educativo de todo el alumnado" (p. 94).

Desde la perspectiva de los autores, la equidad educativa debe promover tres aspectos:

1. Igualdad entre los alumnos en los aspectos, sociales, culturales y económicos que garanticen las condiciones mínimas y necesarias para acceder al servicio educativo

2. Equidad en las medidas destinadas a los estudiantes que contribuyan al logro de los objetivos de aprendizaje.

3. Equidad para la igualdad de potenciales, en el sentido, de que los estudiantes puedan poner en práctica todas sus habilidades para aprender.

Esta aproximación mantiene relación estrecha con la noción de equidad educativa propuesta por Silva (2014, cit. en Priego y Castro, 2021) que se refiere a asegurar

...el acceso, la permanencia y los resultados significativos a todos los miembros de una sociedad evitando la discriminación y atendiendo las desigualdades previas que limitan la capacidad de las personas para aprovechar este bien público (p. 25).

La equidad educativa se conceptualiza en este trabajo como una cualidad orientada a compensar las diferencias sociales, culturales y financieras para el logro de las condiciones de igualdad de oportunidades en: a) el acceso al servicio educativo, b) la permanencia y c) el logro educativo en alumnos de todos los contextos socioculturales; que contribuyan al desarrollo potencial de sus posibilidades de aprendizaje.

En México, la equidad educativa, como mecanismo compensatorio y como aspiración necesaria para la disminución de brechas sociales ha estado presente en el discurso político de las últimas décadas y ha sido impulsada a través de iniciativas promovidas por organismos internacionales.

En materia de legislación, respecto a la equidad se expresa la iniciativa del Estado para implementar medidas que favorezcan el ejercicio pleno del derecho a la educación y a combatir las desigualdades sociales, económicas y regionales (Constitución Política de los Estados Unidos Mexicanos (CPEUM) Art. 3, 2019). Por su parte, la Ley General de Educación refiere la obligación del Estado de prestar los servicios educativos con equidad y excelencia, y algunas acciones que se deberán implementar para tales fines. Además, refiere la necesidad de atender de manera prioritaria a las escuelas de localidades aisladas (Ley General de Educación, 2019, 30 de septiembre).

Por otro lado, el periodo de confinamiento, que trasladó la modalidad presencial a la de distancia, dejó ver que el acceso a las TIC es limitado y representa un problema grave, sobre todo en las localidades con alta dispersión poblacional. El artículo sexto constitucional establece literalmente que:

El Estado garantizará el derecho de acceso a las tecnologías de la información y comunicación, así como a los servicios de radiodifusión y telecomunicaciones, incluido el de banda ancha e internet. Para tales efectos, el Estado establecerá condiciones de competencia efectiva en la prestación de dichos servicios (CPEUM, Art. 6. 15 de mayo del 2019: 12).

Los principios que sustentan la legislación, representan grandes desafíos para el Estado Nacional y en los hechos persisten condiciones de desigualdad que vulneran el derecho de los niños, niñas y adolescentes de recibir una educación de calidad. Como ejemplo, desde el componente de logro educativo, es posible citar los resultados de aprendizaje de la prueba PLANEA (INEE, 2019; INEE, 2018) que ponen en evidencia la brecha en el nivel de logro entre los alumnos que asisten a escuelas ubicadas en entornos favorables, respecto a aquellos que viven en comunidades aisladas y marginadas.

Algunos factores que explican la distancia entre las iniciativas legales y las realidades educativas respecto a la equidad, se relacionan con la ausencia de acciones que garanticen su aplicabilidad (De la Cruz, 2020). En este sentido, a los escenarios de educación rural, se asocian comúnmente problemas relacionados con la pobreza, la 
desnutrición, la falta de acceso a servicios básicos, el trabajo infantil, el analfabetismo, además de la falta de equipamiento y conectividad (INEE, 2019; INEE, 2018; Rockwell y Garay, 2014).

La equidad educativa es concebida también como una condición necesaria desde la perspectiva de los actuales modelos educativos y los fundamentos teóricos que lo sustentan, entendiendo que el proceso de enseñanza debe promover el desarrollo de condiciones que aseguren el tránsito de las diferentes zonas de desarrollo próximo, que en el caso de la modalidad multigrado, se presentan por la mediación de los docentes y de los alumnos cotutores de años más avanzados.

Desde la perspectiva sociocultural, el estudiante juega un papel protagónico en la construcción de aprendizajes. La interacción social con compañeros y con el docente, resulta una herramienta de gran valía para el desarrollo cognitivo y social. Las habilidades y aprendizajes que el estudiante desarrolla en el plano interpersonal, pasan al plano intrapersonal para ser usadas de manera autorregulada (Hernández, 1998).

Desde esta concepción, el alumno se aproxima al conocimiento como sujeto activo, que construye significados sobre lo que aprende no de manera aislada sino como resultado de los procesos de mediación al interior de un contexto específico. Durante los procesos de interacción, se ponen en marcha la expresión, el reconocimiento de puntos de vista, la creación y la resolución de conflictos (Coll, 1997; Hernández, 1998; Baquero, 1997).

En el contexto de la educación formal, el enfoque sociocultural señala que la esencia de las prácticas de enseñanza y el aprendizaje

...reside en las relaciones al interior del llamado triángulo interactivo: la actividad educativa del profesor, las actividades de aprendizaje de los alumnos y el contenido objeto de dicha enseñanza y aprendizaje (Díaz-Barriga, 2006: 14).

La interacción entre el estudiante y el docente o tutor a partir del uso de herramientas es lo que posibilita, el avance gradual hacia la evolución de significados. Entre las funciones centrales del profesor se encuentran la orientación, promoción y guía de la actividad mental constructiva de sus alumnos, a quienes, en el deber ser, brindará una ayuda pedagógica ajustada a su competencia (Coll, 2001 en Díaz-Barriga, 2006).

El análisis de las condiciones desde las cuales se desarrolla la actividad educativa es esencial para valorar si las ayudas pedagógicas que se gestan en los procesos de interacción, se ven desfavorecidas durante el periodo de confinamiento, a través del diseño educativo que el profesor elabora para guiar al estudiante tratando de llevarlo idealmente a tareas de alto nivel cognitivo, bajo las condiciones actuales y con ello determinar los retos y desafíos que implican (Hernández, 1998; Coll, 1997, 2001; Monereo y Castelló, 2001).

\section{Marco metodológico y contexto de investigación}

La aproximación metodológica al contexto se llevó a cabo mediante un estudio de caso de corte hermenéutico, el cual pone énfasis en la singularidad del objeto de estudio y concede especial centralidad a las prácticas de los sujetos de investigación (Cresswell, 1998; Flick, 2015).

\section{Instrumentos de investigación}

La recopilación de evidencia empírica para profundizar en el conocimiento del objeto de interés, se llevó a cabo mediante los siguientes instrumentos de investigación:

a) Búsqueda y selección de información estadística en fuentes oficiales que permitió dimensionar las desigualdades en el acceso a dispositivos y servicios tecnológicos, como principal condicionante para el componente de acceso al servicio educativo en la modalidad a distancia.

b) Diseño, aplicación y análisis de un cuestionario exploratorio a través de Google-forms, para identificar los datos generales de la escuela y de las condiciones de trabajo docente.

c) Diseño, aplicación y análisis de entrevista semiestructurada dirigida al docente a través de la plataforma de video-conferencia Zoom, para caracterizar las dificultades que se presentan para continuar y concluir el ciclo escolar.

Las dimensiones consideradas en el cuestionario aplicado mediante Google-forms fueron las siguientes:

Tabla 1. Dimensiones del Cuestionario

\begin{tabular}{|c|l|}
\hline \multirow{4}{*}{ Docente } & Datos generales \\
\cline { 2 - 2 } & Formación \\
\cline { 2 - 2 } & Experiencia \\
\cline { 2 - 2 } & Condiciones de trabajo docente \\
\hline \multirow{4}{*}{$\begin{array}{c}\text { Contexto social } \\
\text { Organización de las } \\
\text { actividades de } \\
\text { enseñanza y } \\
\text { aprendizaje }\end{array}$} & $\begin{array}{l}\text { Posibilidades de los alumnos para el } \\
\text { acceso a dispositivos tecnológicos y } \\
\text { servicios. }\end{array}$ \\
\cline { 2 - 2 } & $\begin{array}{l}\text { Posibilidades de comunicación } \\
\text { durante la pandemia }\end{array}$ \\
\cline { 2 - 2 } & Planeación de actividades \\
\cline { 2 - 2 } $\begin{array}{c}\text { Componentes de } \\
\text { equidad }\end{array}$ & Evaluación de actividades \\
\cline { 2 - 2 } & Acceso al servicio educativo \\
\cline { 2 - 2 } & Permanencia de la matrícula inscrita \\
\cline { 2 - 2 } & Logro educativo \\
\hline
\end{tabular}

Fuente: Elaboración propia 
En el siguiente cuadro se comparten las dimensiones consideradas en las 3 sesiones de entrevista virtual semiestructurada:

\section{Tabla 2. Dimensiones de la Entrevista}

\begin{tabular}{|c|c|}
\hline Conceptos o proposiciones & Dimensiones \\
\hline $\begin{array}{l}\text { Ayudas pedagógicas: } \\
\text { Ofrecer oportunidades y herramientas para } \\
\text { favorecer el tránsito entre las diferentes } \\
\text { zonas y la internalización de conocimiento. } \\
\text { La participación en la construcción de } \\
\text { aprendizajes, se reduce en la medida en } \\
\text { que los estudiantes se apropian del objeto } \\
\text { de conocimiento. }\end{array}$ & $\begin{array}{l}\text { Ajustes a las } \\
\text { actividades }\end{array}$ \\
\hline Zona de desarrollo próximo: & \\
\hline $\begin{array}{l}\text { La distancia entre el nivel real de } \\
\text { desarrollo, determinado por la capacidad } \\
\text { de resolver independientemente un } \\
\text { problema y el nivel de desarrollo potencial, } \\
\text { determinado a través de la resolución de un } \\
\text { problema bajo la guía de un sujeto más } \\
\text { experimentado. }\end{array}$ & $\begin{array}{l}\text { Procesos de } \\
\text { evaluación } \\
\text { Dificultades para } \\
\text { las actividades } \\
\text { aprendizaje }\end{array}$ \\
\hline Procesos Psicológicos superiores: & \\
\hline $\begin{array}{l}\text { Son humanos en tanto histórica y } \\
\text { socialmente constituidos, son producto de } \\
\text { la línea de desarrollo cultural y presuponen } \\
\text { la existencia de procesos psicológicos } \\
\text { elementales. }\end{array}$ & $\begin{array}{l}\text { Procesos de } \\
\text { interacción }\end{array}$ \\
\hline
\end{tabular}

Fuente: Elaboración con base en Vygotsky, 1934; Coll, 1997 y 2001; Baquero, 1997

El estudio se realizó en una escuela primaria de modalidad unitaria (un docente atiende a los alumnos de primero a sexto grado) ubicada en una comunidad rural del municipio de Singuilucan, Hgo. El docente adscrito a este centro escolar cuenta con estudios de Licenciatura en Ciencias de la Educación y aproximadamente ocho años de experiencia en el trabajo con grupos multigrado. Actualmente, lleva un año y siete meses de trabajo en la institución donde tuvo lugar la presente investigación. La matrícula escolar, está conformada por 18 alumnos distribuidos en los grados de primero a sexto.

\section{Resultados}

El uso de las Tecnologías de la Información y la Comunicación, así como el acceso de servicios básicos como la luz y el teléfono, se han posicionado como elementales dentro de la educación formal bajo la modalidad a distancia, siendo una condicionante para el acceso al servicio educativo en el contexto temporal de la pandemia. Los datos del censo nacional 2020, publicados en la página oficial del Instituto Nacional de Estadística y Geografía (INEGI), permiten dimensionar la precariedad en el acceso a servicios y dispositivos tecnológicos en el contexto de estudio.

En éste, los datos señalan que, a nivel nacional, $37.6 \%$ de la población cuenta con equipo de cómputo, mientras que
87.5\% con celular. En el estado de Hidalgo, sólo 30\% de la población tiene computadora, aunque $85 \%$ cuenta con teléfono móvil. En el escenario particular del municipio de Singuilucan, sólo $13.2 \%$ de la población cuenta con computadora y $85 \%$ con celular (INEGI, 2020).

La cobertura de red eléctrica tiene un alcance importante a nivel nacional, estatal y municipal, pero no sucede así con el acceso a internet. Mientras que a nivel Nacional alrededor de la mitad de la población cuenta con servicio de internet, el porcentaje se reduce a $38.8 \%$ en el estado y a $15.4 \%$ a nivel municipal (INEGI, 2020).

En el ámbito local, apenas el 50\% de la población total tiene acceso al agua potable (INEGI, 2020). El servicio del vital líquido representa serias limitaciones en la localidad de estudio, lo que deriva en escasas posibilidades de cumplir en los hogares con las medidas de salubridad indispensables como el lavado de manos, situación que debe ser valorada en el planteamiento de la estrategia para el regreso a clases presenciales.

Respecto a los servicios, la totalidad de los estudiantes tiene red eléctrica y sólo la mitad de ellos cuenta con agua potable en su domicilio. Apenas $5 \%$ de los estudiantes tiene acceso a internet, lo que en números representa un alumno. Esta situación deja ver que si bien en la comunidad hay infraestructura tecnológica para acceder a redes de internet, la limitante radica en las pocas posibilidades de sostenimiento económico del servicio para las familias.

Ante las limitantes de comunicación, el docente entrevistado ha optado por el diseño de fichas de trabajo impresas que son entregadas a los padres de familia mediante visitas escolares. La planeación de las actividades en periodo de confinamiento es por grado escolar. Es decir, seis planeaciones diferentes, con actividades según las asignaturas y contenidos enmarcados en los programas de estudio. Este panorama deja ver la compleja situación que enfrentan los docentes de escuelas rurales de modalidad multigrado en periodos de confinamiento, pues se redoblan esfuerzos en el trabajo referido a la planeación, entrega y revisión de materiales de manera física para seis grados diferentes.

Por otro lado, el docente refiere que los materiales otorgados por la Secretaría de Educación Pública, por medio del programa Aprende en Casa, son poco útiles, ya que las actividades contenidas en las fichas didácticas son poco pertinentes para el contexto social de los estudiantes, además de que tienen escaso acceso a dispositivos para la sintonización de los programas televisivos.

Otra limitante, según reporta el docente, está relacionada a la escasa prioridad que las familias otorgan a las actividades educativas, pues los estudiantes se involucran en la dinámica de los quehaceres del hogar o de las 
actividades del campo, dedicando tiempo insuficiente a las tareas propuestas por el docente.

Asimismo, una restricción importante respecto a la comunicación con los padres de familia, es la escasez de señal telefónica, pues en la localidad la cobertura del servicio, llega únicamente a lugares específicos. Por tal hecho, el docente sólo establece contacto con un padre de familia que le informa sobre las dificultades de su hijo y de algunos estudiantes vecinos. Estas circunstancias derivan en la acumulación de tareas, dudas sin resolver por parte de los alumnos y procesos de evaluación parciales y a destiempo.

Por otro lado, los padres y madres de familia y/o tutores disponen de poco tiempo para acompañar los procesos de aprendizaje de sus hijos como resultado de las actividades económicas que desempeñan. Además, la mitad de los padres y madres de familia sólo tiene estudios concluidos de primaria, lo que dificulta el tratamiento didáctico de algunos contenidos a desarrollar por parte de los alumnos.

Con la información presentada y a la luz del enfoque de equidad, es posible analizar las dificultades de determinadas poblaciones de alumnos, respecto a tres componentes: 1) el acceso, 2) la permanencia y 3) el logro educativo.

\section{1) Acceso}

Respecto al acceso, México había alcanzado en las últimas décadas la cobertura casi total del servicio educativo para la población en edad escolar del nivel primaria (INEE, 2019; Juárez y Juárez, 2007). Sin embargo, es importante actualizar datos estadísticos e impulsar proyectos de investigación centrados en el estudio de las posibilidades de ingreso al servicio educativo bajo los efectos de la pandemia.

\section{2) Permanencia}

Si bien en la escuela estudiada no se reportan bajas escolares, a nivel nacional, el componente de permanencia representa ya serios problemas, pues según un informe publicado por el INEGI (2021), al menos 1.9\% de la población de estudiantes en edad primaria no concluyó el ciclo escolar 2019-2020 y 217,000 alumnos no se inscribieron al periodo 2020-2021. Estos datos, representan un retroceso de años en cuanto a la permanencia en el sistema educativo de los alumnos en edad escolar primaria.

\section{2) Logro educativo}

El logro educativo, se visualiza como el componente más afectado durante el periodo de confinamiento. Los resultados de ésta y otras investigaciones que le anteceden, indican que las posibilidades de aprendizaje de los estudiantes están limitadas por la precariedad de servicios, las particularidades de las familias y la poca posibilidad de comunicación con el docente para la resolución de dudas y la socialización de los aprendizajes. Además, esta situación se explica en alguna medida por la ausencia de los procesos de mediación inherentes a la modalidad presencial (Gutiérrez, 2020).

Antes de la pandemia, los resultados de las pruebas de aprendizaje, como PLANEA, indican que al menos 50\% de los estudiantes del nivel primaria no lograban alcanzar los aprendizajes mínimos para desenvolverse en el mundo actual (INEE, 2019). Los alumnos con los resultados más bajos, viven en localidades indígenas, rurales y urbano-marginales. Esta situación, aunada a los nuevos retos que representa la pandemia por COVID, a corto, mediano y largo plazo estará también asociada al incremento de rezago educativo.

Ante esta realidad, las ventajas del multigrado referidas por el docente y otros investigadores se ven limitadas por las pocas posibilidades de encuentro y socialización del aprendizaje entre los diferentes actores.

Desde la perspectiva sociocultural, "el aprendizaje se sitúa en un contexto determinado; depende de las oportunidades y restricciones que éste ofrece" (Díaz Barriga, 2006:18). En este sentido, las características del contexto de la educación rural evidencian que la precariedad de condiciones sociales y económicas ofrecen limitadas posibilidades de aprendizaje de los estudiantes, asociadas a las dificultades de comunicación con el docente, el acompañamiento de los padres de familia, los recursos y materiales para el aprendizaje, así como la incorporación de los alumnos en las actividades laborales y del hogar.

Por otro lado, el apoyo pedagógico, brindado a los estudiantes durante su proceso de aprendizaje, se reduce a la participación casi exclusiva del padre de familia, y las ayudas pedagógicas que brinda el docente y los compañeros de grupo, se ven desfavorecidas.

En las actividades de evaluación, descritas por el docente para valorar los productos de aprendizaje de los estudiantes, se identifica un proceso característico de la evaluación desde la perspectiva sociocultural, desde el cual, la evaluación, además de valorar el nivel de desarrollo real (diagnóstico), determina el nivel de desarrollo que pueden llegar a alcanzar de manera próxima mediante la prestación de ciertas ayudas pedagógicas (Hernández, 1998).

En el caso de la educación a distancia, la evaluación de las actividades y el acompañamiento que de ésta se deriva, son diferentes para cada alumno y dependen de sus posibilidades de comunicación. Por ejemplo, para los alumnos a quienes les resulta complejo comunicarse vía telefónica o por WhatsApp, deben esperar las fechas de entrega de los productos escritos, evaluados por parte del 
docente, mismos que integran algunas observaciones, recomendaciones y en ocasiones, valoraciones cuantitativas. El periodo en el que los alumnos y padres de familia tienen posibilidad de tener contacto con el docente, va desde los 15 días hasta un mes.

Las pocas posibilidades de interacción entre alumnos y docentes, las debilitadas ayudas pedagógicas que reciben los estudiantes, la escasez de materiales educativos y los procesos de evaluación a destiempo, no llevan a los alumnos al desarrollo de Proceso Psicológicos Superiores Avanzados que requieren la mediación cultural y social.

Los resultados de investigación se pueden resumir en la siguiente tabla, acorde a las dimensiones valoradas en las entrevistas:

Tabla 3. Resultados por dimensión de análisis

\begin{tabular}{|c|c|}
\hline $\begin{array}{l}\text { Conceptos y } \\
\text { proposiciones }\end{array}$ & Resultados \\
\hline $\begin{array}{c}\text { Ayudas } \\
\text { pedagógicas }\end{array}$ & $\begin{array}{l}\text { - Las ayudas pedagógicas se ven } \\
\text { desfavorecidas ante la ausencia de co- } \\
\text { tutorías, socialización de experiencias, } \\
\text { aprendizajes y acompañamiento. } \\
\text { - Los materiales y recursos de } \\
\text { aprendizaje fueron escasos. }\end{array}$ \\
\hline $\begin{array}{l}\text { Zona de desarrollo } \\
\text { próximo }\end{array}$ & $\begin{array}{l}\text { - Hay dificultades en la aplicación del } \\
\text { proceso de evaluación diagnóstica y } \\
\text { formativa que limitan la identificación } \\
\text { del estado de aprendizaje de los } \\
\text { estudiantes y las posibilidades de } \\
\text { tránsito entre las diferentes zonas de } \\
\text { desarrollo. } \\
\text { - Se identifica que, el seguimiento al } \\
\text { proceso de aprendizaje está } \\
\text { condicionado por el acceso a formas de } \\
\text { comunicación remota con el docente. }\end{array}$ \\
\hline $\begin{array}{l}\text { Procesos } \\
\text { Psicológicos } \\
\text { superiores }\end{array}$ & $\begin{array}{l}\text { - La escasa mediación cultural y social, } \\
\text { restringe el desarrollo de Procesos } \\
\text { Psicológicos Superiores. } \\
\text { - La participación de los estudiantes en } \\
\text { las actividades del hogar limita el } \\
\text { tiempo destinado a las tareas } \\
\text { académicas y las posibilidades de } \\
\text { significación social y cultural de los } \\
\text { contenidos escolares. }\end{array}$ \\
\hline
\end{tabular}

Fuente: Elaboración propia

\section{Conclusiones}

La actual pandemia pone en evidencia que no han sido compensadas las desigualdades sociales, económicas, familiares y escolares, así como las posibilidades de acceso a servicios y dispositivos tecnológicos. Desde esta perspectiva, la equidad se limita a un discurso político que se pone en tela de juicio al evidenciar la poca capacidad y voluntad de respuesta del sistema educativo para atender las necesidades de la heterogeneidad de contextos que existen en el territorio nacional.
Por otro lado, además del acceso, la permanencia y el logro educativo, desde la perspectiva de SánchezSantamaría y Ballester (2013), es importante que la equidad educativa ofrezca a los alumnos la posibilidad de desarrollar potencialmente sus posibilidades de aprendizaje: alcance reducido en los espacios rurales por los condicionantes sociales, económicos y familiares.

Los resultados de esta investigación invitan a poner atención en la figura docente, pues las aspiraciones por la equidad y la excelencia educativa deben reconocer su labor para la mejora del sistema educativo. En este sentido, es importante avanzar en el conocimiento desde la investigación educativa sobre los procesos de formación inicial y continua, el acompañamiento, la pertinencia de los contenidos programáticos y los materiales educativos, la formación tecnológica, los ajustes a las políticas de gestión y de dirección en pequeños centros escolares, así como ofrecer iniciativas que promuevan su autonomía pedagógica.

Es necesario reconocer que, las acciones en materia de equidad, deben superar el ámbito escolar para situarse también en el político, social, cultural y económico, ya que la escuela tiene una capacidad limitada de ofrecer condiciones de equidad y justicia, comparada con el peso de los factores sociales y culturales para el desarrollo de una educación de calidad (De la Cruz, 2016; Blanco, 2013).

A partir de los resultados, se propone accionar políticas que favorezcan la dotación de dispositivos tecnológicos a alumnos y docentes de todas las escuelas del país, la ampliación en la cobertura de la señal telefónica y redes de internet, la formación de docentes y alumnos en el desarrollo de competencias tecnológicas, pero sobre todo, la puesta en marcha de políticas económicas y sociales que avancen hacia una distribución justa de los recursos y hacia la promoción de medidas compensatorias en los contextos más desfavorecidos.

Todo ello, entendiendo que la equidad educativa promueve condiciones propicias para el diseño educativo que el docente desarrolla para la aplicación de los principios de los modelos educativos y perspectivas, principalmente, con orientación socio constructivistas, que sustentan la educación básica, los cuales requieren de las mínimas condiciones mencionadas para poder verse realizados.

Se reconoce que el estudio de las perspectivas de otros actores educativos, como alumnos, padres de familia, personal de la supervisión y autoridades educativas, es de importante relevancia para construir un escenario más completo de la actividad educativa actual. Por lo que se sugiere realizar estudios que recuperen sus experiencias para la construcción de conocimiento de la realidad social en este complejo periodo de pandemia. 
Por otro lado, es importante mencionar que, a pesar de la falta de infraestructura, equipamiento y conectividad, se requiere la inventiva de los docentes para poner en acción estrategias innovadoras y creativas que les permitan ofrecer ayudas pedagógicas viables de acuerdo con los recursos disponibles. Los mecanismos de influencia que configuran los docentes a partir del diseño de las actividades, las evaluaciones diagnósticas, formativas y los materiales didácticos que diseñan, son claves para asegurar que el estudiante construya significados (Vygotsky, 1934; Coll, 1997, 2001; Baquero, 1997). Las metodologías de enseñanza fundamentadas en el socio constructivismo ofrecen a los estudiantes amplias posibilidades de significar lo escolar en lo cotidiano y superar algunas dificultades presentadas en el componente de logro educativo.

Finalmente, es importante señalar que, para identificar la zona de desarrollo próximo del estudiante y se puedan adecuar actividades y ofrecer ayudas pedagógicas que le permitan aprender, es necesario que el docente cuente con suficiente información educativa de sus estudiantes y del contexto social y cultural en el que se desenvuelven, para proponer tareas con un alto nivel cognitivo que favorezcan la construcción de conocimientos.

\section{Referencias}

Acero, Oscar, Briceño, Angélica, Orduz, Marcela y Tuay, Rosa Nidia (2020). Realidades de la educación rural en Colombia, en tiempos del COVID-19. Centro de recursos para el aprendizaje y la educación. Universidad Santo Tomás. https://repository.usta.edu.co/handle/11634/32325 [23 abril de 2021]

Aguilera, Ma Antonieta, Quezada Steffania y Camacho, Kenya Ruth (2019). "Diagnóstico de las condiciones de las escuelas multigrado" en: Sylvia Schmelkes y Guadalupe Aguila (Eds.) La educación multigrado en México. pp. 75-106. Instituto Nacional para la Evaluación de la Educación. https://www.inee.edu.mx/wpcontent/uploads/2019/01/La-Educacio\% CC\%81n-

Multigrado_BIS.pdf [23 abril de 2021]

Amador, Rocío (2020). Aprende en casa con sana distancia en tiempos de COVID-19. En Instituto de Investigaciones sobre la Universidad y la Educación [iisue] (2020), Educación y pandemia. Una visión académica. Universidad Nacional Autónoma de México. [En línea] pp.138-144 https://www.iisue.unam.mx/nosotros/covid/educacion-ypandemia [27 abril de 2021]

Arteaga, Paola (2011). Los saberes docentes de maestros en primarias con grupos multigrado. [En línea] http://rededucacionrural.mx/lineas-deinvestigacion/formacion-docente-inicial/los-saberes-docentes-demaestros-en-primarias-con-grupos-multigrado/ [27 abril de 2021]

Baquero, Ricardo. (1997) Vygostsky y el aprendizaje escolar. Aique grupo editor.

Blanco, Emilio (2013). Los límites de la escuela. Educación, desigualdad y aprendizajes en México. El Colegio de México.

Bustos, Antonio. (2010). Aproximación a las aulas de escuela rural: Heterogeneidad y aprendizaje en los grupos multigrado. Revista de Educación, 373-378. http://www.revistaeducacion.educacion.es/re352/re352_16.pdf $\quad[14$ abril de 2021]

Carla, Tatiane, Ramos, Eliane y Montanari, Rafael (2020). Dificultades de la educación remota en las escuelas rurales del norte de Minas Gerais durante la pandemia de Covid-19. Research, Society and Development,
Vol.
9 ,
núm
8 ,
pp.
$1-14$

https://rsdjournal.org/index.php/rsd/article/download/6053/5829/1001 14 [3 mayo de 2021]

Coll, Cesar (1997) Aprendizaje escolar y construcción del conocimiento. Paidós.

Coll, Cesar (2001). Constructivismo y educación: la concepción constructivista de la enseñanza y el aprendizaje. En Cesar Coll, Jesús Palacios y Alvaro Marches (comps.), Desarrollo psicológico y educación. Tomo 1: Psicología de la educación escolar (157-190). Madrid, España: Alianza.

Coll, Cesar y Monereo, Carles (2008). Psicología de la educación virtual: Aprender y enseñar con las tecnologías. Morata.

Constitución Política de los Estados Unidos Mexicanos [Const.] Art. 6. 11 de diciembre del 2013

Constitución Política de los Estados Unidos Mexicanos [Const.] Art. 3. 15 de mayo del 2019.

Creswell, John W. (1998) Qualitative inquiry and research design. Choosing among five traditions. Sage Publications, Inc.

De la Cruz, Gabriela (2017). Igualdad y equidad en educación: retos para una América Latina en transición. Educación. Vol. 26, núm 51, pp. 159-178. https://red.pucp.edu.pe/ridei/files/2018/05/180501.docx_.pdf [26 abril de 2021]

Delgado, Gabriela (2020). Igualdad educativa y postpandemia. En Instituto de Investigaciones sobre la Universidad y la Educación [iisue] (2020), Educación y pandemia. Una visión académica. Universidad Nacional Autónoma de México. [En línea]. pp. 183-194. https://www.iisue.unam.mx/nosotros/covid/educacion-y-pandemia [24 abril de 2021]

Díaz Barriga, Frida. (2006). Enseñanza situada. Vínculo entre la escuela y la vida. McGraw Hill.

Didriksson, Axel (2020). Ante la pandemia, evitar reproducir la desigualdad social y educativa. En Instituto de Investigaciones sobre la Universidad y la Educación [iisue] (2020), Educación y pandemia. Una visión académica. Universidad Nacional Autónoma de México. [En línea]. pp. 154-163. https://www.iisue.unam.mx/nosotros/covid/educacion-y-pandemia [28 abril de 2021]

Estrada, María del Mar (2015). Multigrado en derecho propio. Revista latinoamericana de estudios educativos. Vol. 45, núm. 2, pp. 43-62. https://www.cee.edu.mx/rlee/revista/r2011 2020/r_texto/t 2015 2 031.pdf [23 abril de 2021]

Flick, Uwe (2015). El diseño de investigación cualitativa. Madrid. Morata.

Gutiérrez, Norma Georgina (2020). Aprende en casa. Propuesta para la educación básica en México en tiempos de COVID-19. Notas de Coyuntura del Centro Regional de Investigaciones Multidisciplinarias. núm. 29, pp. 1-6. https://doi.org/10.22201/crim.001r.2020.29 [2 mayo de 2021]

Hernández, Gerardo (1998). Paradigmas en psicología de la educación. Paidós educador.

Hurtado, Frank Junior (2020) La educación en tiempos de pandemia: los desafíos de la escuela del siglo XXI. CIEG, Revista Arbitrada del Centro de Investigación y Estudios Gerenciales. núm. 44, pp. 176-187.

Instituto Nacional para la Evaluación Educativa (2018). Informe de resultados. [En línea] https://www.inee.edu.mx/wpcontent/uploads/2018/12/P1I244.pdf [2 mayo de 2021]

Instituto Nacional para la Evaluación Educativa (2019) Informe de Resultados. [En línea] https://www.inee.edu.mx/wpcontent/uploads/2019/04/P1I245.pdf [24 abril de 2021]

Instituto Nacional de Estadística y Geografía (24 de marzo del 2021). Panorama Sociodemográfico de México. [En línea] https://www.inegi.org.mx/programas/ccpv/2020/tableros/panorama/ [19 abril de 2021]

Instituto Nacional de Estadística y Geografía (2021). Encuesta para la medición del impacto COVID-19 en la educación (ECOVID-ED). Presentación de resultados. [En línea] https://www.inegi.org.mx/contenidos/investigacion/ecovided/2020/do c/ecovid ed 2020_presentacion_resultados.pdf [23 abril de 2021]

Juarez, Diego (2017). Percepciones de docentes rurales multigrado en México y El Salvador. Sinéctica. Revista Electrónica de Educación. 
núm. 49. pp. 1-16. http://www.scielo.org.mx/pdf/sine/n49/2007-7033sine-49-00002.pdf [6 mayo de 2021]

Juárez, Diego y Rodríguez, Carlos Rafael (2016). Factores que afectan la equidad educativa en escuelas rurales en México. Pensamiento educativo. Revista de Investigación Educacional Latinoamérica. Vol. 53, núm. $\quad 2, \quad$ pp. $\quad 1-15$. https://www.researchgate.net/publication/309785269 Factores que a fectan_a_la_equidad_educativa_en_escuelas_rurales_de_Mexico $[28$ abril de 2021]

Ley General de Educación 9/2019. Diario Oficial de la Federación 4-5, 30 de septiembre del 2013. [En línea] http://www.diputados.gob.mx/LeyesBiblio/pdf/LGE 300919.pdf [27 abril de 2021]

Loyd, Marion (2020) Desigualdades educativas y la brecha digital en tiempos de COVID-19. En Instituto de Investigaciones sobre la Universidad y la Educación [iisue] (2020), Educación y pandemia. Una visión académica. Universidad Nacional Autónoma de México. [En línea] pp.115-121 https://www.iisue.unam.mx/nosotros/covid/educacion-y-pandemia [24 abril de 2021]

Mérida, Yliana y Acuña, Luis Alan (2020). Covid-19, Pobreza y Educación en Chiapas: Análisis a los Programas Educativos Emergentes. Revista Internacional de Educación para la Justicia $\begin{array}{llllll}\text { Social. Vol. 9, núm. 3, pp. 61-82. } & \end{array}$ https://doi.org/10.15366/riejs2020.9.3.004 [21 abril de 2021]

Narváez, Daniela Alejandra y Yépez, Jenny Katherine (2021). Tiempos de pandemia y el papel de la familia en la educación. Revista Huellas. Vol. 1 núm. $13, \quad$ pp. 10-16. https://revistas.udenar.edu.co/index.php/rhuellas/article/view/6304 [3 mayo de 2021]

Priego, Lenin Bruno y Castro, Mariana (2021). Equidad y escuelas multigrado ¿ruptura o continuidad de la política educativa? Revista Latinoamericana de Estudios Educativos. Vol. 51, núm. 1, pp. 177204. https://doi.org/10.48102/rlee.2021.51.1.267 [7 mayo de 2021]

Rockwell, Elsie y Garay, Claudia (2014). Las escuelas unitarias en México en perspectiva histórica: Un reto aún vigente. Revista Mexicana de Historia de la Educación. Vol. 2, núm. 3, pp. 1-24. http://www.rmhe.somehide.org/index.php/revista/article/view/33/43 [11 mayo de 2021]

Sánchez-Santamaría, José y Ballester, María Gracia (2013). Desarrollando el éxito educativo para todos: reflexiones, propuestas y retos conceptuales en torno a la equidad educativa. REICE. Revista Iberoamericana sobre Calidad, Eficacia y Cambio en Educación. Vol. 12, núm. $2, \quad$ pp. https://repositorio.uam.es/handle/10486/661497 [21 abril de 2021]

Santos, Limber (2011). Aulas multigrado y circulación de los saberes: Especificidades didácticas de la escuela rural. Profesorado: Revista de curriculum y formación del profesorado, 15(2), 71-91. https://www.redalyc.org/pdf/567/56719129006.pdf $[13$ marzo de 2021]

Trejo-Quintana, Janneth (2020) La falta de acceso y aprovechamiento de los medios y las tecnologías: dos deudas de la educación en México. En Instituto de Investigaciones sobre la Universidad y la Educación [IISUE] (2020), Educación y pandemia. Una visión académica. Universidad Nacional Autónoma de México. [En línea] pp.122-129 https://www.iisue.unam.mx/nosotros/covid/educacion-y-pandemia [24 abril de 2021]

Vivanco-Saraguro, Ángel (2020) Teleducación en tiempos de COVID-19: brechas de desigualdad. CienciAmérica. Vol. 9, núm 2, pp. 166-175. http://cienciamerica.uti.edu.ec/openjournal/index.php/uti/article/view/ 307 [19 abril de 2021]

Weiss, Eduardo (2000) La situación de la enseñanza multigrado en México. Perfiles educativos. Vol. XXII, núm. 89-90, pp. 57-76. http://www.scielo.org.mx/pdf/peredu/v22n89-90/v22n89_90a4.pdf [25 abril de 2021]

Vygotsky, Lev. (1934). Pensamiento y lenguaje. Teoría del desarrollo cultural de las funciones psíquicas. Ediciones Fausto.
Vygotsky, Lev. (2009) El desarrollo de los procesos psicológicos superiores. Debolsillo. 